Supporting Information

\title{
Gold-Incorporated Cobalt Phosphide Nanoparticles on Nitrogen-Doped Carbon for Enhanced Hydrogen Evolution Electrocatalysis
}

Xiaoyan Wang, ${ }^{\dagger}$ Yang Fei, ${ }^{\dagger}$ Wei Li,${ }^{\dagger}$ Lingya $Y_{i},{ }^{\dagger}$ Bomin Feng, ${ }^{\dagger}$ Yixiang Pan,${ }^{\dagger}$ Weihua Hu, ${ }^{*, \dagger}$ and Chang Ming Li;,;,s

† Institute for Clean Energy and Advanced Materials, School of Materials \& Energy, Southwest University; Chongqing Key Laboratory for Advanced Materials and Technologies of Clean Energies, 2 Rd. Tiansheng, Beibei, Chongqing 400715, China

$\ddagger$ The State Key Lab of Polymer Materials Engineering, Polymer Research Institute of Sichuan University, Chengdu 610065, China

$\S$ Institute of Materials Science \& Devices, Suzhou University of Science and Technology, Suzhou 215009, China.

* Corresponding author. E-mail: whhu@swu.edu.cn (W. H. Hu); ecmli@swu.edu.cn (C. M. Li). 


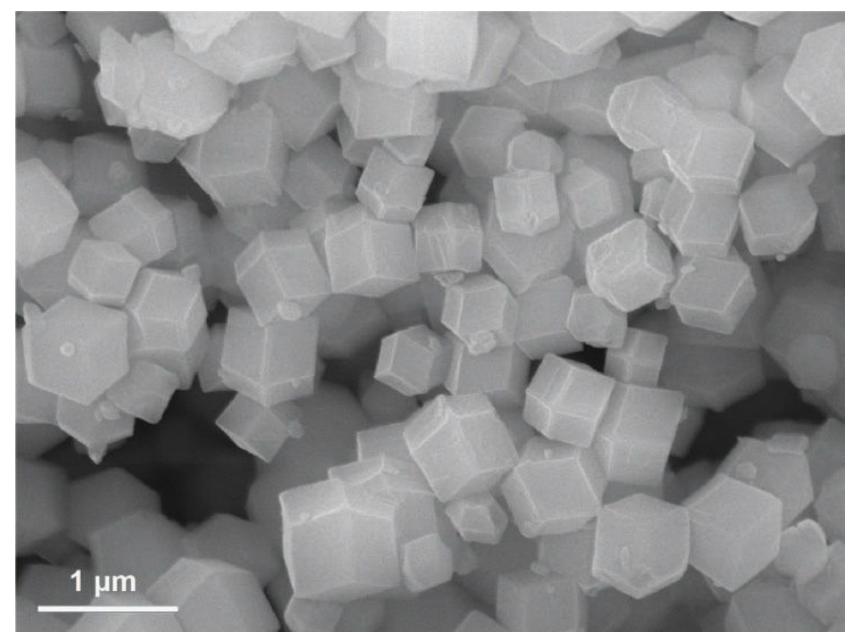

Figure S1. SEM image of ZIF-67.

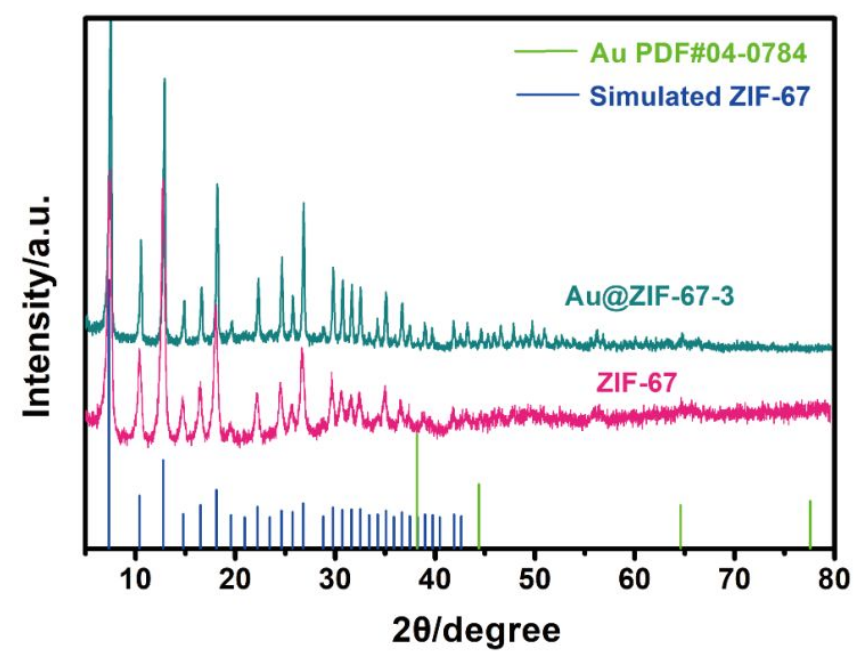

Figure S2. XRD patterns of Au@ZIF-67-3 and ZIF-67.

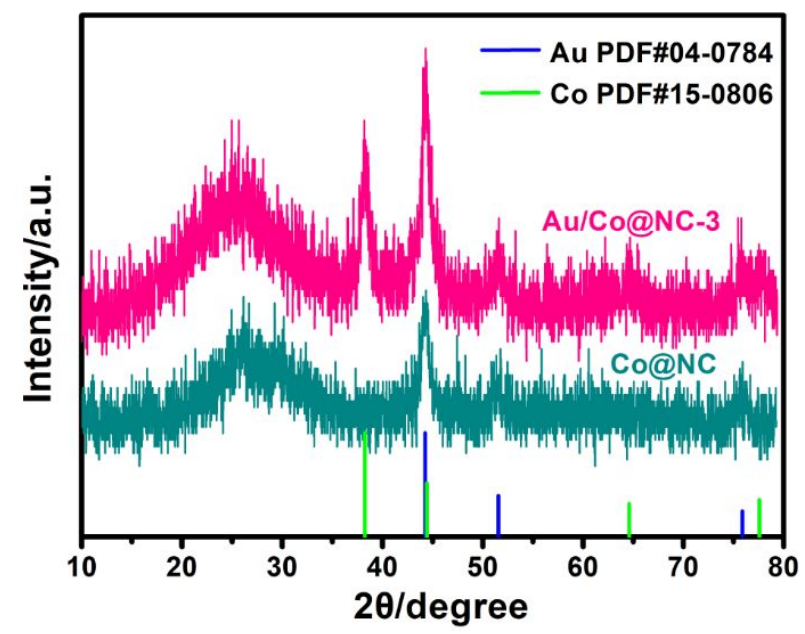


Figure S3. XRD patterns of Au/Co@NC-3 and Co@NC.

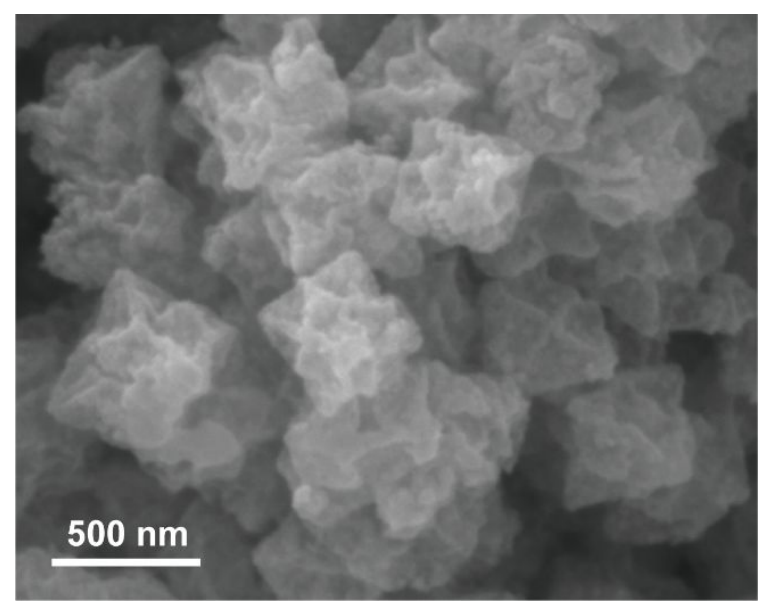

Figure S4. SEM image of Au/Co@NC-3.

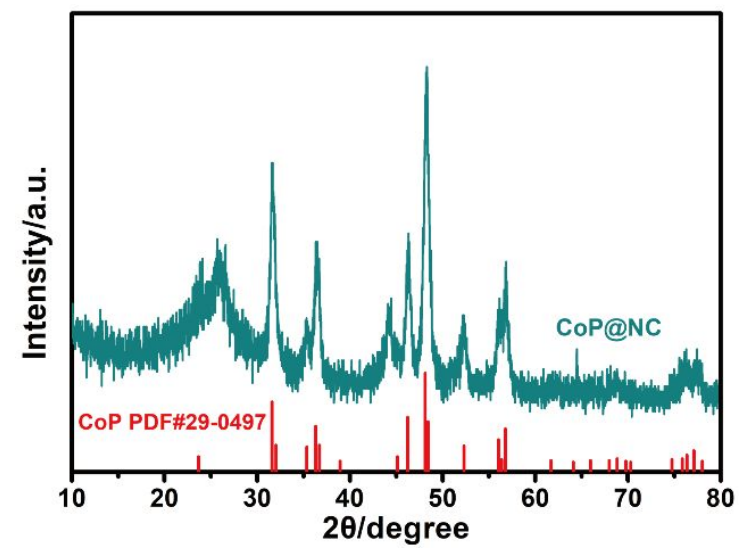

Figure S5. XRD pattern of CoP@NC. 


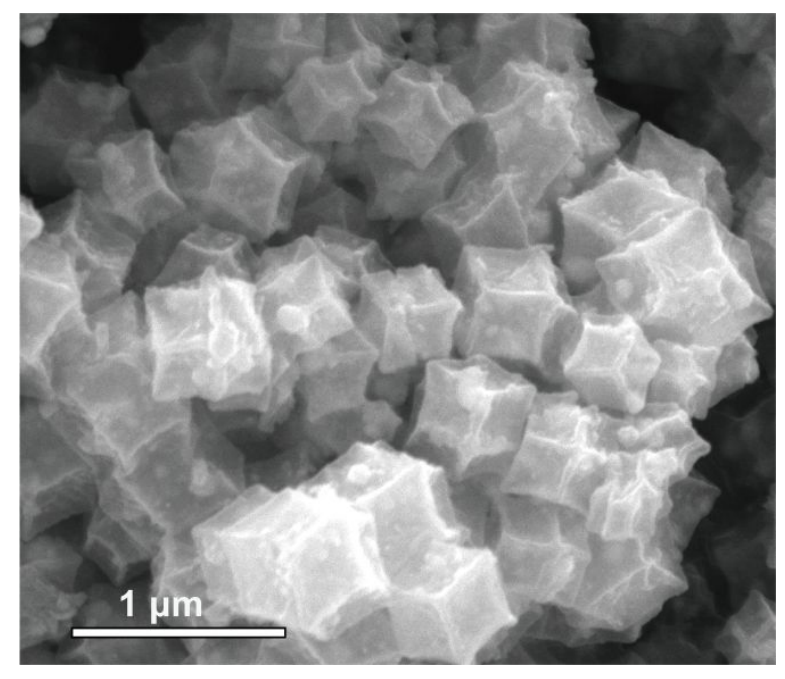

Figure S6. SEM image of Co@NC.

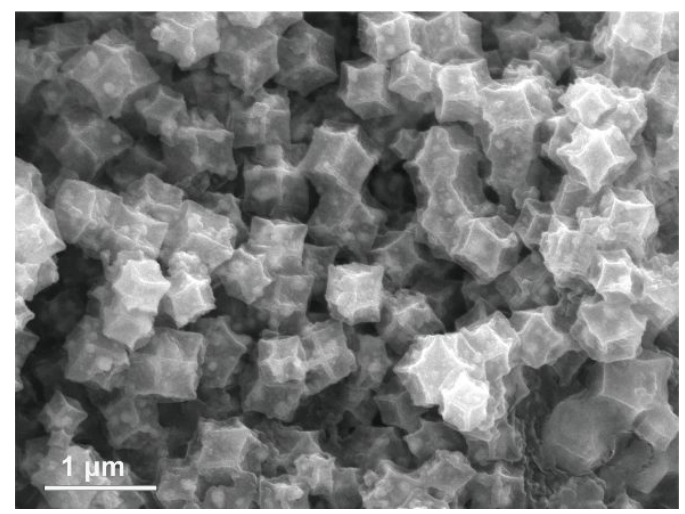

Figure S7. SEM image of CoP@NC. 


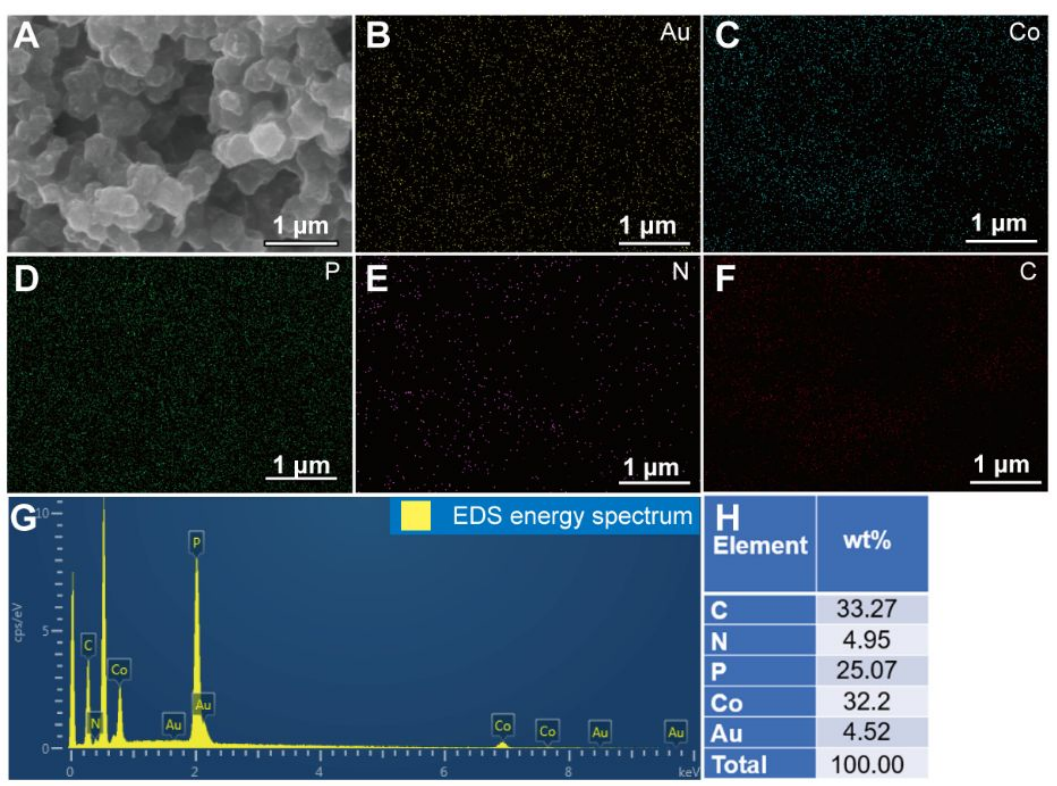

Figure S8. SEM image (A), EDS elemental mapping images of Au (B), Co (C), P (D), $\mathrm{N}(\mathrm{E}), \mathrm{C}(\mathrm{F})$, EDS spectrum $(\mathrm{G})$ and element content table $(\mathrm{H})$ of $\mathrm{Au} / \mathrm{CoP} @ \mathrm{NC}-3$.
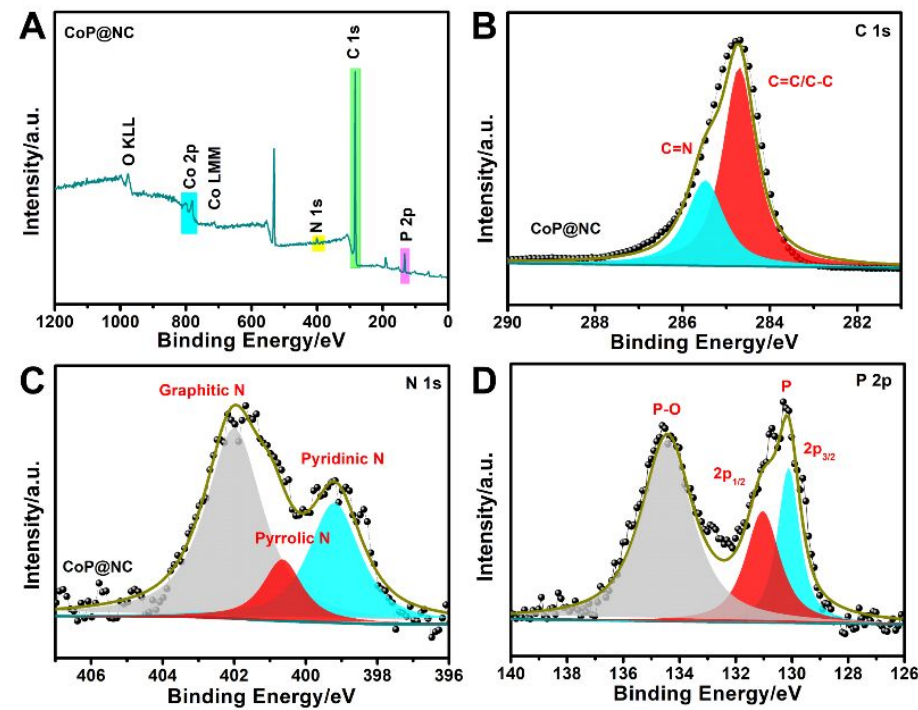

Figure S9. XPS survey (A), C 1s (B), N 1s (C) and P 2p spectra (D) of CoP@NC. 

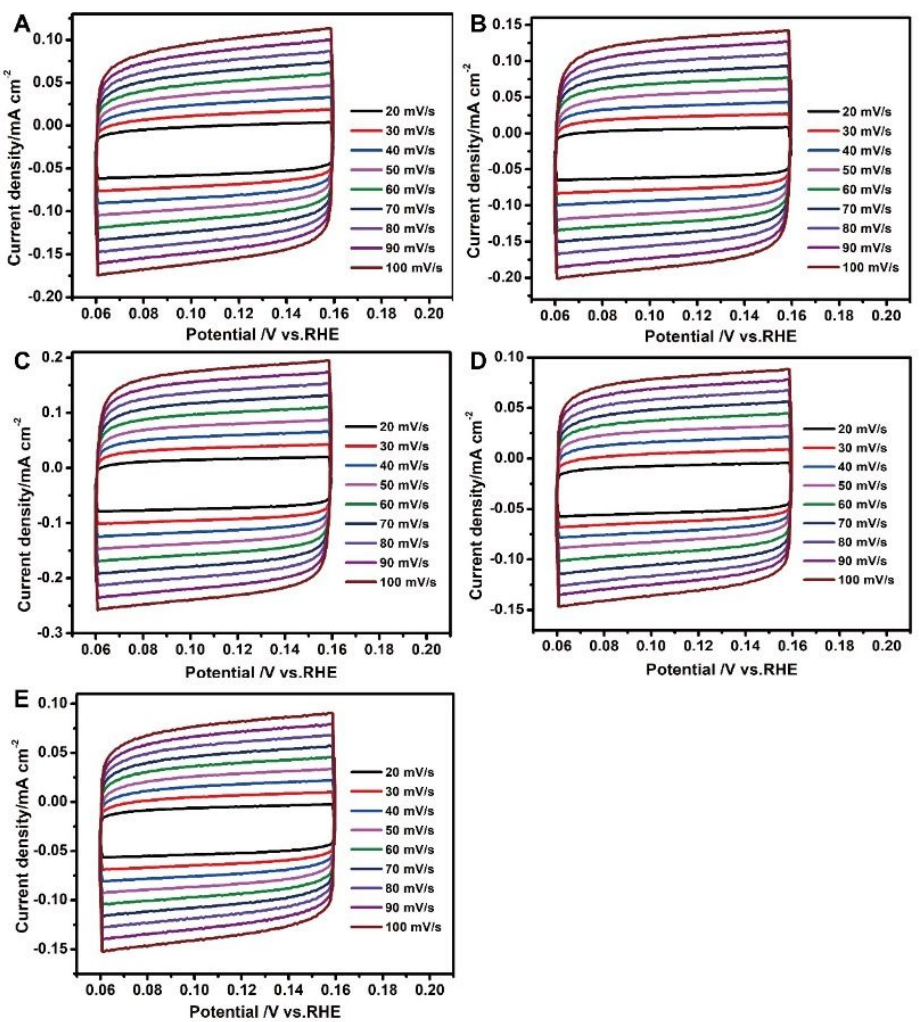

Potential $N$ vs.RHE

Figure S10. Scan rate dependence of the current densities in the CV curves of different HER catalysts with scan rates ranging from $20 \mathrm{mV} \mathrm{s}^{-1}$ to $100 \mathrm{mV} \mathrm{s}^{-1}$ at intervals of $10 \mathrm{mV} \cdot \mathrm{s}^{-1}$. (A) Au/CoP@NC-1, (B) Au/CoP@NC-2, (C) Au/CoP@NC-3, (D) Au/CoP@NC-4, (E) CoP@NC.

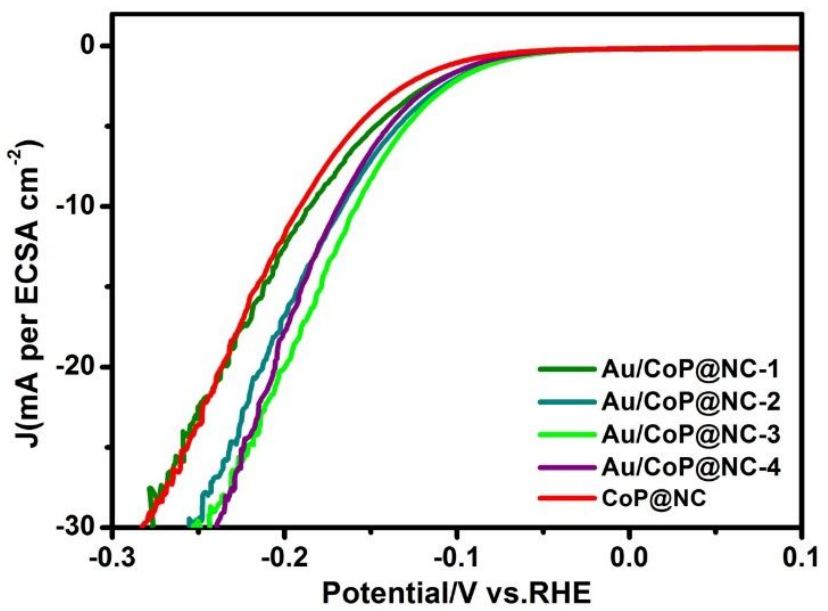

Figure S11. ECSA-normalized HER polarization curves of various electrocatalysts in $1 \mathrm{M} \mathrm{KOH}$. 